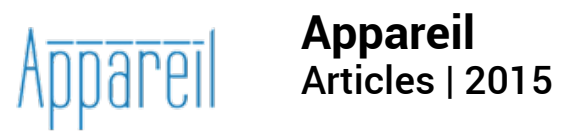

\title{
Le musée, c'est la laïcité en acte
}

\section{Jean-Louis Déotte}

\section{OpenEdition}

\section{Journals}

\section{Édition électronique}

URL : http://journals.openedition.org/appareil/2170

DOI : 10.4000/appareil. 2170

ISSN : 2101-0714

Éditeur

MSH Paris Nord

\section{Référence électronique}

Jean-Louis Déotte, «Le musée, c'est la laïcité en acte », Appareil [En ligne], Articles, mis en ligne le 08 juin 2015, consulté le 30 juillet 2020. URL : http://journals.openedition.org/appareil/2170 ; DOI : https://doi.org/10.4000/appareil.2170

Ce document a été généré automatiquement le 30 juillet 2020.

\section{(c) (i) (9)}

Appareil est mis à disposition selon les termes de la Licence Creative Commons Attribution - Pas d'Utilisation Commerciale - Pas de Modification 4.0 International. 


\title{
Le musée, c'est la laïcité en acte
}

\author{
Jean-Louis Déotte
}

\section{NOTE DE L'ÉDITEUR}

Conférence donnée à Santiago, $1^{\mathrm{er}}$ colloque organisé par l'Université catholique du Chili dans le cadre de la Bibliothèque nationale portant sur musées et patrimoine, avril 2015 sous la responsabilité du professeur Gonzalo Leiva de l'Université catholique du Chili.

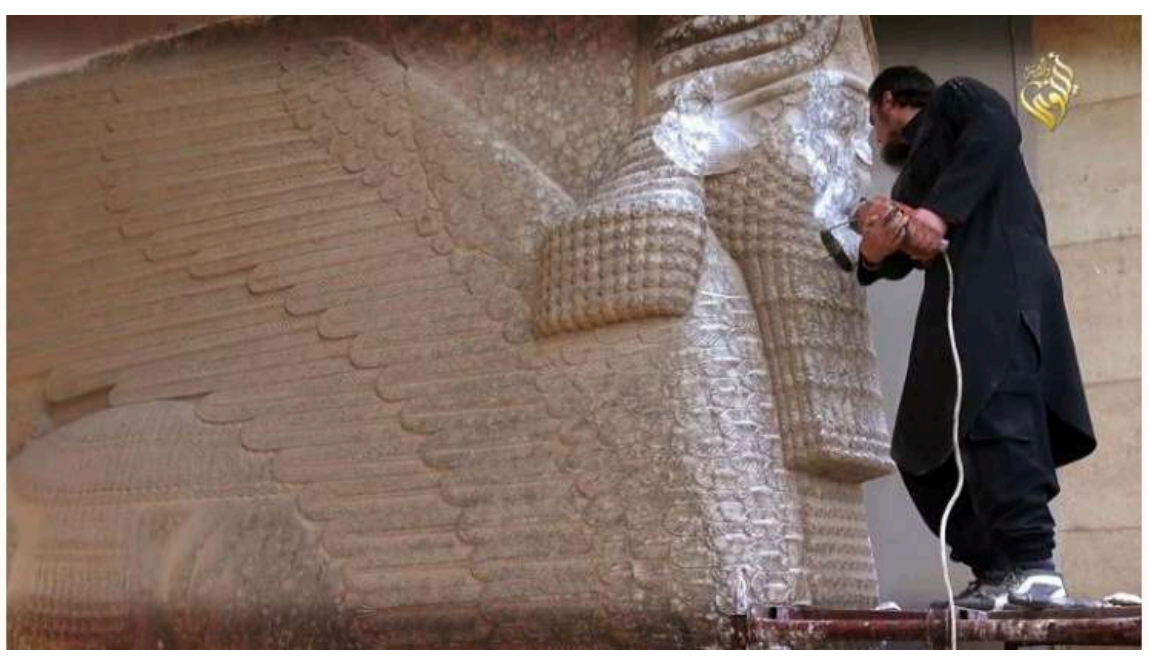

Des militants du groupe El défigurent un taureau ailé assyrien sur un site archéologique de Mossoul, dans le nord de l'Irak

1 Nous partirons de la situation créée par l'État islamiste (EI) dans les zones qu'il contrôle en Irak, la destruction du site d'Hatra, mais aussi des attentats perpétrés contre le musée du Bardo à Tunis en mars 2015 et évidemment contre ses visiteurs cosmopolites. À ces actions, il faudrait ajouter la destruction des statues géantes de Bouddha à Bàmiyan en Afghanistan en 2001 par des talibans.

2 Nous avons assisté à des actions anti-muséales et donc anti-patrimoniales dans d'autres parties du monde : par exemple les revendications couronnées de succès des Kanaks de 
Nouvelle-Calédonie française pour récupérer ce qu'ils considéraient comme leurs divinités ${ }^{1}$, alors que selon les critères de l'UNESCO, c'étaient des suspens, c'est-à-dire des œuvres dont l'existence est indissociable de celle du musée. Appelons suspens, des œuvres de culte, qui ont donc eu une destination religieuse et communautaire et qui, par suite de leur accaparement lors d'actions coloniales d'annexion ou comme butins de guerre révolutionnaire, se retrouvèrent ainsi privées de toute capacité de générer une communauté. Elles produisent en revanche un public, comme celui qui fut abattu à l'entrée du Bardo à Tunis. L'existence de ce public, au contraire d'une communauté, ne dépend pas de l'existence de l'œuvre.

3 Il en va de même dans l'actuelle Russie, où l'Église orthodoxe fait pression sur les institutions culturelles pour se réapproprier les grandes icônes ${ }^{2}$. Aux USA, dans les États du Sud, les communautés amérindiennes négocient avec les conservateurs de musées qui détiennent leurs totems, pour pouvoir à certaines dates de l'année, organiser des rituels en leur présence. Le musée, fermé au public, devient ainsi pour quelques jours un lieu de culte pour des communautés dont l'identité est indissociable de ces artefacts ${ }^{3}$.

4 Ce n'est que pour les Occidentaux, depuis le $\mathrm{xvIII}^{\mathrm{e}}$ siècle, que la stricte relation esthétique, universalisable sans concept, au sens de Kant, est indissociable du musée, qui est cet appareil spécial qui dissocie l'œuvre de sa destination cultuelle, la livrant à l'histoire de l'art et aux risques sans limites de l'exposition esthétique pour un public toujours indéterminé.

5 La preuve historique d'une telle universalisation du jugement esthétique depuis lors peut être apportée après la destruction des statues assyriennes de Mossoul, laquelle a immédiatement soulevé l'indignation générale et un projet de musée virtuel est en train de voir le jour :

C'est une initiative culturelle spontanée, mondiale et fulgurante, à l'image $d u \mathrm{xxI}^{\mathrm{e}}$ siècle numérique. Le "projet Mossoul» est un musée virtuel en construction pour réagir à la barbarie des destructions d'œuvres d'art assyrien du musée irakien de Mossoul. Ce projet en 3D, lancé en ligne le 8 mars, en appelle à la foule des internautes. Il s'agit d'obtenir tout autant des images des œuvres qui étaient exposées au musée que de l'aide en ligne provenant d'experts en patrimoine ou en programmation informatique. Depuis le lancement, l'audience et les contributions extérieures ne cessent de grimper sur le site grâce à un marketing viral planétaire. Matthew Vincent et Chance Coughenour, sont les deux archéologues à l'origine de cette opération. ${ }^{4}$

6 La simultanéité des attaques de Mossoul et de Tunis nous conduit à remettre en cause notre croyance, issue des Lumières, dans l'irréversibilité de «l'éducation esthétique de l'homme ", pour reprendre le titre de l'œuvre de Schiller. Voire dans la croyance plus fondamentale en un art et un patrimoine universels. Ce qui surgit au contraire, c'est l'expression de profonds différends (Lyotard) entre des régimes du symbolique ou entre des rapports à la loi, irréconciliables. Par loi, il ne faut pas entendre seulement l'aspect juridique et axiologique, mais plus fondamentalement le rapport d'imitation du cosmos, ce qui inclut le rapport au savoir et à l'apparaître des choses comme des communautés. C'est la dimension du paraître, de l'apparence, de la nécessité pour chacun et pour chaque collectif de donner à voir le meilleur de lui-même qui nous fera donner à la notion de cosmétique plus d'importance qu'à celle d'ontologie. Cosmétique donc : à partir de l'acception grecque du kosmos : l'ordre parfait des groupes fonde ce qui est bel et bon sur le modèle de l'ordre des étoiles. À l'évidence, pour les djihadistes 
de Mossoul, les statues des rois assyriens du musée appartenaient à un autre cosmos que celui des Lumières occidentales, dès lors ces témoignages de pierre étaient une injure à la loi révélée du Coran. Le djihadisme actuel n'est qu'un courant parmi d'autres de l'Islam, qui prône un retour radical à l'origine prophétique en interprétant selon la lettre les sourates et non selon l'«esprit». Au contraire, les voix qui s'élèvent aujourd'hui dans et hors l'Islam pour le "moderniser", vont dans le sens d'une spiritualisation.

7 Si le monde païen occidental put être accommodant envers les divinités orientales comme le montre pour exemple la coexistence dans la Rome impériale d'une multitude de temples, c'était que le principe de l'édification d'un culte et sa fréquentation résidait dans l'utilité que l'évergète pouvait en attendre. Ainsi le succès du christianisme et son adoption progressive dans l'Empire ne tiennent qu'au choix privé de l'empereur Constantin. Selon P. Veyne ${ }^{5}$, le nouveau culte va s'imposer par une sorte de mimétisme social, de conformisme, et non parce que l'esclavage ou les combats du cirque seraient devenus pour la plupart des Romains insupportables. Le passage d'une cosmétique à une autre peut être, dans un premier temps au moins, indolore. D'ailleurs, on ne peut parler de différend entre cosmétiques qu'à partir du moment où la nouvelle cosmétique, dorénavant d'essence "révélée", par le devenir livre de la loi ("inlibration») ou par l'incarnation de l'infini dans le fini, impose à l'ancienne, devenue « idolâtre » et "païenne », une soumission, puis une destruction, qui la rend muette peu à peu. Le « génie du Christianisme » (Chateaubriand) s'est ainsi imposé au " génie du paganisme » (M. Augé).

8 C'est aujourd'hui, au Proche et au Moyen-Orient, la répartition étatique voulue par les Français et les Britanniques sur fond de disparition de l'Empire ottoman (accord secret Sykes-Picot du 16 mai 1916) qui s'effondre. Et les anciennes idoles, qui selon Malraux auraient dû se métamorphoser en sculptures, redeviennent ce qu'elles n'avaient jamais cessé d'être pour les musulmans intégristes. Comme l'écrivait Benjamin, avec l'« ultramodernité » (la mondialisation), c'est l'archaïque (la préhistoire ${ }^{6}$ : Urgeschichte) qui remonte à la surface. Ce qui implique que ce qui revient, ne revient pas au même, mais avec des « habits neufs ", ici ceux de la mondialisation. Ce qui permet de comprendre à la fois la destruction des idoles préislamiques et sa diffusion sur Internet. Dès lors, ce renouveau de l'iconoclasme, inauguré historiquement par l'incarnation chrétienne aussi bien à Byzance qu'avec la Réforme ${ }^{7}$, ne doit pas nous transformer à notre tour en iconodules. Car il y a au préalable un lien entre la foi et les technologies de communication, qu'il faut interroger8. Il est évident que la diffusion de ces images de destruction, comme celle des exécutions d'Occidentaux, comme les appels à la guerre sainte montés selon le modèle des films hollywoodiens, prouvent que l'utilisation de l'image ne fait pas nécessairement problème à ceux qui pourtant détruisent les œuvres muséales qui sont néanmoins, à leur manière, des images de ces dieux qu'elles ont été par ailleurs.

9 Les islamistes n'ont aucun problème avec les images des média (les vidéos privées ou propagandistes de Ben Laden le prouvent), mais ne supportent pas les ruines patrimoniales, parfaitement inoffensives en apparence, des statues et des monuments assyriens ou bouddhistes. C'est que le musée et le patrimoine en général ne sont pas des moyens de communication, des média, mais des appareils. Il faudra donc distinguer deux statuts de l'image : le résultat plastique de l'appareillage de tel ou tel art (c'est le cas des suspens où un art, par exemple chrétien, s'est trouvé appareillé par le musée). 
Dès lors la circulation de ces images est secondaire; et des images qui n'ont été produites que pour être échangées, c'est le cas des photos numériques produites par des téléphones portables type smartphone. Les œuvres des musées sont aujourd'hui soumises à ce second usage, comme si la conservation des collections n'était plus l'office muséal par excellence, mais la communication des selfies.

À ce différend cosmétique, il faut en ajouter un autre, car entre inlibration musulmane et incarnation chrétienne, il y avait un socle commun : le rapport à la loi est régi dans les deux cas par la norme de la révélation, et d'ailleurs de nombreux accommodements furent possibles, comme le montre H. Belting', telle l'intégration comme décor en Occident de l'ornementation géométrique musulmane ou des images des miniatures dans la sphère d'influence du chi'isme, en Perse.

11 Cet autre différend cosmétique, plus fondamental, concerne la norme de révélation de la loi et la norme projective qui surgit, tout armée, à Florence au $\mathrm{Xv}^{\mathrm{e}}$ siècle avec Brunelleschi, Masaccio, puis Alberti, avec l'invention de l'appareil perspectif. La géométrie musulmane était plane (pavage du plan), l'occidentale introduisit la tridimensionnalité qui rend possible la représentation. Depuis lors, la cosmétique occidentale est projective, tous les appareils qui l'appliquent sont projectifs, même s'ils sont d'origine arabe comme la camera obscura. Cette capacité de tracer sur un support idéalisé comme transparent ce qui se projette de là-bas, devant la fenêtre du plan de projection, et avance vers le spectateur pour laisser son empreinte, est la clef du dessin entendu par les Traités renaissants comme designo. Le designo synthétise alors des traits hétérogènes: c'est un projet d'avenir, un relevé de l'Antique, un moyen de transmission pour l'architecte, la notation d'une idée, etc. C'est l'ensemble de la civilisation occidentale qui va être bouleversé, à commencer par la politique (Machiavel), mais aussi bien la réalité économique: la monnaie comme moyen d'échange entre marchandises concrètes va devenir crédit, c'est-à-dire achat du temps lui-même. Le capitalisme ne suppose pas seulement une ascèse mondaine où la réussite d'un travail est la preuve de l'élection divine, selon les analyses classiques de M. Weber, mais plus fondamentalement un ethos où le bien-être de tous et de chacun réside dans la capacité de se projeter dans le futur. Ceux qui ne peuvent plus se projeter sont condamnés au pire destin: l'éternel retour du même, la dépression. La temporalité selon les Lumières était orientée selon un principe qualitatif : le progrès supposant un accomplissement de l'idéalité, la temporalité post-moderne, démocrate-capitaliste, ne connaît que l'accroissement quantitatif : le développement sans limites. C'est, nous dit Benjamin $^{10}$, une religion sans Dieu, mais non pas sans cultes, où il n'y a ni dogme spécifique ni théologie. "La durée du culte est permanente. Le capitalisme est la célébration d'un culte sans trêve et sans merci »... Ce culte est culpabilisant et non expiatoire. A contrario, la temporalité des religions révélées est cyclique.

Politiquement, le différend entre l'Islam et la démocratie classiques ne relève pas d'une "guerre des religions ", auquel cas les démocrates depuis la Grèce antique seraient des croisés, mais d'une confrontation entre des modes d'énonciation de la loi. Cette loi est " hétéronome " dans le cas des religions révélées, "autonome » depuis la Grèce homérique, au sens où le foyer du sens se trouve idéalement « au milieu » des hommes dans une démocratie ${ }^{11}$, en une place vide qu'aucun pouvoir ne saurait occuper définitivement. C'est un espace de délibération qui s'ouvre sur l'impossibilité d'une incarnation ou d'une incorporation, dès lors les moyens comme les fins de l'action commune échappent à la soumission qui est au cœur de l'Islam. Il n'y aura bientôt plus 
de frontières au politique, puisque ce qui définissait le "propre » d'une communauté s'est effacé. L'entretien devient infini : il ne s'agit plus de commenter indéfiniment le texte sacré, mais d'accueillir par l'image diagrammatique des projections de ce que l'on place en face de soi pour l'étudier.

13 C'est ce vide que la religion qui ressurgit après les révolutions démocratiques des «printemps arabes » ne peut supporter : elle a gagné le combat contre les dieux païens en imposant la figure de l'Unicité, mais se trouve désormais devant une absence de

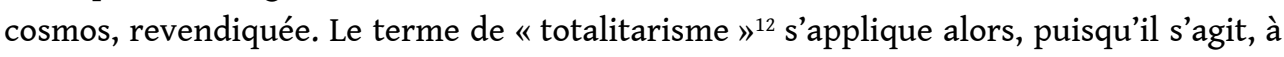
partir d'un calife, de reconstituer du corps communautaire partout où des musulmans sont présents.

14 C'est parce que musée et patrimoine sont d'essence démocratique que les djihadistes, qui veulent rétablir un califat, c'est-à-dire un certain mode du théologico-politique comme foyer du sens, sont dans l'obligation de détruire ce qui, comme le musée, le patrimoine ou l'écriture de l'histoire, est d'essence projective.

15 Mais le musée et le patrimoine surgirent eux aussi de l'épreuve de l'angoisse du vide en répondant à une autre orientation que celle de la perspective qui se proposait pour tâche de donner des traces d'un monde perdu : la Nature. La distance constitutive de la projection perspective entre l'écran où s'écrivent les traces de ce qui est là-bas, devant l'écran, et la Nature devenue un objet rationnel, cette distance est devenue abyssale et la réponse, historique en raison de la Révolution française. Dès lors, le musée a été amené à tordre le temps par une sorte de rétroaction, une projection vers le passé. Il a commencé à accueillir ce qui avait été, souvent condamné à l'obscurité de ce qui était sacré, dans les couvents, les églises et les cours princières. Puis, il a été le lieu d'épanouissement d'un art strictement esthétique, avec Manet, les impressionnistes, Cézanne, etc.

16 Le moteur de son extension a résidé dans l'épreuve du maintenant ${ }^{13}$ : ce sont les œuvres de l'art contemporain qui entraînent le collectionneur-conservateur à sauver dans le passé des archives des réserves, l'Autrefois (das Gewesene) pour reprendre une expression de Benjamin. C'est-à-dire selon le modèle photographique qui structure sa conception de la temporalité : ce qui gît dans le passé sans y avoir été réellement inscrit, mais nous attend comme le ferait une pellicule photosensible non développée. Ce n'est donc pas par hasard si ces deux appareils, le musée, la photo, ont surgi à peu près en même temps, comme les deux faces d'une même époque de la norme projective : au moment de la Révolution française qui brise le lien avec le théologicopolitique chrétien. Alors que la nation ne peut plus s'incarner dans le corps immortel du roi, la France invente le rôle politique du musée et du patrimoine (Quatremère de Quincy). C'est en effet la même assemblée politique (la Convention) qui va décréter la mort du roi et le sauvetage du patrimoine tout en dissolvant les communautés d'essence religieuse, comme la communauté juive, réduite depuis lors à un ensemble de citoyens, chacun étant libre d'exercer sa religion selon le principe énoncé par le royaliste constitutionnaliste Stanislas de Clermont-Tonnerre : « il faut tout refuser aux juifs comme Nation, il faut tout accorder aux juifs comme individus » (4 août 1789). ${ }^{14}$

17 C'est là le fondement de la laïcité à la française : le musée, parce qu'il n'est pas communautaire, est essentiellement laïc. C'est un appareil politico-esthétique. On ne voit d'ailleurs pas en quoi la même politique ne pourrait être appliquée à la « communauté » musulmane. 

virtuelles les œuvres détruites comme une volonté de renouer avec un acte fondateur sur le plan politico-esthétique. À la différence près que c'est une nouvelle fantasmagorie qui se développera, une sorte de nouvelle Alexandrie numérique, car les Ptolémées avaient eu le projet de réunir à Alexandrie non pas les sculptures grecques, mais la plus grande bibliothèque possible, dans un endroit agréable, un jardin, le Mouséion.

21 La numérisation universelle des œuvres achèvera donc le musée imaginaire de Malraux qui deviendra une bibliothèque, non pas de photos, mais de spectres digitaux. Car inévitablement, c'est la relation esthétique qui changera de nature.

\section{BIBLIOGRAPHIE}

Belting Hans, Florence et Bagdad. Une histoire du regard entre Orient et Occident, traduction N. Ghermani et A. Rieber, Paris, Gallimard, coll. « Le temps des images », 2012 [2008].

Benjamin Walter, « Le capitalisme comme religion », in Fragments, Paris, PUF, 2001.

Christin Olivier, Une révolution symbolique. L'iconoclasme huguenot et la reconstruction catholique, Paris, Éditions de Minuit, 1991.

Déotte Jean-Louis, Oubliez ! Les ruines, l'Europe, le musée, Paris, L'Harmattan, 1995, 326 p.

Déotte Jean-Louis, L'Homme de verre. Esthétiques benjaminiennes, Paris, L'Harmattan, 1998.

Déotte Jean-Louis, L'époque des appareils, Paris, Leo Scheer, 2004.

Douglas Mary, De la souillure. Essai sur les notions de pollution et de tabou, Paris, La Découverte, 2005, $205 \mathrm{p}$. 
Lefort Claude, Un homme en trop. Réflexions sur l'Archipel du Goulag, Paris, Seuil, 1976.

Selbach Gerard, Les musées d'art américains : une industrie culturelle, Paris, L'Harmattan, 2000.

Veyne Paul, Quand notre monde est devenu chrétien (312-394), Paris, Albin Michel, 2007.

\section{NOTES}

1. Les Kanaks de Nouvelle-Calédonie ont subi pendant des décennies le système colonial français, lequel s'exerçait au nom de leur prétendue émancipation, jusqu'au jour où l'esprit de communauté païen l'a emporté sur les principes de la République et qu'à partir du massacre d'Ouvéa commandité par la droite (Pons, Chirac) (1988), il a bien fallu que le socialiste M. Rocard reconnaisse la spécificité irréductible de l'ethos kanak.

2. La controverse entre l'Église et les musées était très violente il y a vingt ans, aujourd'hui elle continue, mais d'une façon moins visible. L'argument de l'Église est le suivant: les musées possèdent les objets «volés " pendant la Révolution. De plus, les objets de cultes doivent servir comme objets de culte. L'argument du Musée : ces objets ont été sauvés contre la destruction pendant l'époque soviétique. Il y a beaucoup de bâtiments (monastères, etc.) d'où les gens d'Église ont chassé les conservateurs des musées. Il y a des cas où les icônes passent du musée à une église. Dans certains cas, ces icônes sont très anciennes : par exemple deux icônes du XIV siècle (disparues par la suite) et une du XII ${ }^{\mathrm{e}}$ siècle (cette dernière est conservée dans de très bonnes conditions "muséales »). L'Église est une institution très influente aujourd'hui, et les musées cherchent un compromis (les directeurs de l'Ermitage et du Musée Russe affirment que «l'Église et le musée ne sont pas ennemisé : parfois, ils prêtent leurs pièces à l'Église [il y a des cas de non-retour] et organisent des liturgies dans les Églises qui gardent encore leur statut de musée [comme la Cathédrale St-Isaac à Saint-Pétersbourg]. Parfois, au lieu de retourner un original, on propose une copie. En 2010, on a adopté une loi [qui a des formules assez vagues d'ailleurs] qui prescrit le retour des œuvres à l'Église ", informations : Denis Skopin).

3. Gérard Selbach, Les musées d'art américains : une industrie culturelle, Paris, L'Harmattan, 2000.

4. En savoir plus sur http://www.lemonde.fr/arts/article/2015/03/17/projet-mossoul-un-museevirtuel-pour-reagir-face-a-la-barbarie-de-l-etat-

islamique_4595546_1655012.html\#Yb1P1U6DAfeRYfLq.99

5. Paul Veyne, Quand notre monde est devenu chrétien (312-394), Paris, Albin Michel, 2007.

6. Comme je l'ai montré dans L'Homme de verre. Esthétiques benjaminiennes (Paris, L'Harmattan, 1998), la plupart des notions concernant la temporalité se dédoublent chez W. Benjamin. Ainsi la "préhistoire» peut être rattachée dans d'autres textes aux "phénomènes originaires » goethéens.

7. Olivier Christin, Une révolution symbolique. L'iconoclasme huguenot et la reconstruction catholique, Paris, Éditions de Minuit, 1991. La violence djihadiste n'a rien à envier à l'iconoclasme huguenot !

8. Je renvoie ici aux réflexions de Derrida sur foi et «télécommunications ».

9. Hans Belting, Florence et Bagdad. Une histoire du regard entre Orient et Occident, traduction

N. Ghermani et A Rieber, Paris, Gallimard, 2012 [2008].

10. Walter Benjamin, «Le capitalisme comme religion », in Fragments, Paris, PUF, 2001, p. 110 : «Ce que le capitalisme a d'historiquement inouï tient à ce que la religion est non plus réforme mais ruine de l'être. »

11. Je reprends ici la notion de « démocratie » aux travaux de Claude Lefort, laquelle est chez lui un mode de légitimité, alors qu'un Lyotard n'y verrait qu'un mode de domination, lui préférant la notion de « république ».

12. Au sens de Claude Lefort, Un homme en trop. Réflexions sur l'Archipel du Goulag, Paris, Seuil, 1976. Le terme de "fascisme djihadiste" utilisé par Badiou est un contre-sens, car il ramène un 
phénomène nouveau, essentiellement théologico-politique, à un épisode contre-révolutionnaire moderne.

13. Au sens du « Maintenant de la connaissabilité » (Erkennbarkeit) chez Benjamin.

14. Il faut se souvenir que Marx dans la Question juive va précisément attaquer l'« homme » des droits de l'homme sur ce point : l'individualisme bourgeois comme atome solitaire.

15. Jean-Louis Déotte, Oubliez! Les ruines, l'Europe, le musée, Paris, L'Harmattan, 1995, 326 p.; L'époque des appareils, Paris, Leo Scheer, 2004.

16. Mary Douglas, De la souillure. Essai sur les notions de pollution et de tabou, Paris, La Découverte, 2005, 205 p.

\section{RÉSUMÉS}

Les attaques diffusées sur Internet par «l'État islamique » contre les œuvres muséales, contre le patrimoine anté-islamique, contre le tourisme, rappellent que les liens entre la foi et les télécommunications sont indissociables. Elles apportent la preuve que le musée n'est pas un médium de communication mais un appareil qui a changé la nature des œuvres, qui auparavant étaient de destination, cultuelle et communautaire, pour devenir des suspens, livrés à l'exposition. Le musée creuse une sorte d'espace vide au cœur du symbolique et du théologicopolitique : là où le pouvoir était incarné, le surgissement de l'esthétique artistique livre la société à une indétermination fondamentale. Cette indifférence quant aux destinations cultuelles ouvre l'espace de la laïcité politique et rend possible un art qui est par essence anarchique, acosmique.

\section{INDEX}

Mots-clés : laïcité, musée, islamisme iconoclaste 